\title{
Erratum to: Study of the Microarc Oxidation of Aluminum Modified with Silicon Carbide Particles
}

\author{
M. A. Markov $a$, S. N. Perevislov ${ }^{b}$, A. V. Krasikov ${ }^{a}$, D. A. Gerashchenkov ${ }^{a}$, \\ A. D. Bykova ${ }^{a}$, and M. L. Fedoseev $a$ \\ a NRC "Kurchatov Institute," Central Research Institute of Structural Materials "Prometey," \\ ul. Shpalernaya 49, St. Petersburg, 191015 Russia \\ ${ }^{b}$ St. Petersburg State Technological Institute (Technical University), St. Petersburg, 190013 Russia \\ e-mail: barca0688@mail.ru
}

Received December 27, 2019

DOI: $10.1134 /$ S1070427219120277

In the title section in the authors list Previslov should be replaced by Perevislov.

The original article can be found online at https://doi.org/10.1134/S107042721804002X 